\title{
Double Layer Metamaterial Superstrate for Performance Enhancement of a Microstrip Patch Antenna
}

\author{
F.Z.Khoutar ${ }^{1}$, M. Aznabet ${ }^{1}$, O. EL Mrabet ${ }^{1}$, O. Nayat-Ali ${ }^{1}$ \\ \{fatikhoutar@gmail.com¹, mariamaznabet@gmail.com ${ }^{1}$, o.mrabet@gmail.com ${ }^{1}$ \} \\ Information and Telecommunication system laboratory ${ }^{1}$, Abdelmalek Essaadi \\ University,Tetuan,Morocco,fs.tetouan.contact@gmail.com
}

\begin{abstract}
In this paper, a metamaterial antenna operating at a frequency of $5.8 \mathrm{GHz}$ is presented. Metamaterial superstrate cover acts as a lens which focuses the EM radiations due to the unusual properties of the metamaterial.This leads to improvement performance of patch antenna along with its physical protection. The proposed metamaterial lens consists of a double layer of 9-by-9 matrix of Split-Ring Resonators (SRRs), and is positioned above a probe-fed rectangular patch resonating at $5.8 \mathrm{GHz}$. The structure improve the gain and directivity of a simple patch antenna up to $7 \mathrm{~dB}$. Simulation results of the proposed antenna are presented and discussed in this letter.
\end{abstract}

Keywords: Metamaterial (MTM),Split Ring Resonator (SRR).

\section{Introduction}

An enormous growth in the field of telecommunication has generated enormous needs and has resulted in a major technological change at the antenna level, depending on the transmission frequency, data rate or transmission range.

The antenna is one of the most critical components in the wireless communication systems, since the performance of the antenna alone can seriously influence the overall performance of the entire system. The design goal of an antenna is often governed by the specifications provided by the customer. Many applications specify that the antenna must be conformal, and that it should have a high directivity along a direction when transmitting. These requirements, namely high directivity is the major developing trends among antennas and always presents a great challenge to engineers working in this field. The traditional antenna technology to achieve the high directivity needs multiple radiating elements to fabricate the antenna array [1]. However, such array design requires a complex feed network, and the demands that the multiple antenna elements be maintained properly. This is because the malfunction of one or more antenna elements may sometimes seriously affect the overall performance of the antenna system, Other interesting solutions to enhance the directivity of the patch antenna have been suggested: the first one was to make use of a DGS technique to modify the parameter of antenna and the second one proposed recently was to sandwich the antenna by dielectric layers of the same permittivity [2] [3]. 
More recently, another approach is proposed in order to improve the directivity of the antenna, which is based on the use of artificial materials such as left- handed metamaterials [4]. These new kinds of artificial materials, constructed from periodic unit structures that are compactly crowded into an effective material, open the way to observe some exotic properties that are impossible to achieve with natural materials.

Many research works have been achieved in obtaining the high directivity of antenna by using metamaterial structures [5-12]. MTMs can be fabricated through various ways such as photonic crystal [9], electromagnetic band gap (EBG) structure, frequency selective surface (FSS) [1011], and other periodic artificial material which can be designed to have a low/zero refractive index [12-13].Particularly, negative refractive index zero or near zero index metamaterial, and furthermore lead to potential applications, many of which were considered difficult in the past. These include, for example, perfect lensing [14], invisibility cloaking [15] and miniaturizing microwave devices [16].

In this paper, we presented a metamaterial antenna for WLAN application operating at the frequency of $5.8 \mathrm{GHz}$. The double layer metamaterial superstrate used in this design was constructed by split ring resonator (SRRs), introduced for the first time by Pendry et al. in 1999 [17] to achieve a negative permeability in a certain frequency range. The main attractive feature of this element is its ability to exhibit a quasi-static resonant frequency at wavelengths that are much larger than its own size. We have investigated the effect of metamaterials Radom on the directivity, gain and bandwidth of the antenna by using CST Microwave studio. It is found that by optimizing the separation distance between the antenna and the double layer metamaterial superstrate the directivity of the antenna has been increased by $7 \mathrm{~dB}$.

\section{Metamaterial antenna design}

\subsection{Presentation of Metamaterial antenna}

Metamaterials are typically engineered by arranging a set of small scatterers or apertures in a regular array throughout a region of space, thus obtaining some desirable bulk electromagnetic behavior. The desired property is often one that is not normally found naturally (negative refractive index, near-zero index, etc.). Over the past ten years, metamaterials have moved from being simply a theoretical concept to a field with developed and marketed applications. Three dimensional metamaterials can be extended by arranging electrically small scatterers or holes into a two-dimensional pattern at a surface or interface. This surface version of a metamaterial has been given the name metasurface For many applications, metasurfaces can be used in place of metamaterials. Metasurfaces have the advantage of taking up less physical space than do full three-dimensional metamaterial structures,Metamaterial Antenna is a class of antennas that use metamaterial to miniaturize antenna systems and improve their performance. Their purpose, as for any electromagnetic radiated element, is to propagate energy into free space. It consists of an MTM superstrate cover above a reference element.

The operation of the MTM superstrate is explained where the scattered radiations from the patch are concentrated due to the MTM superstrate cover above. This function increases the gain and directivity of the patch antenna.

Moreover, this type of antenna with metamaterial layer is called resonant cavity antenna that generally consists of one or more surfaces Metamaterial, a ground plane and a source of excitation placed inside the cavity. The different planes are separated by the cavity height $(\mathrm{H})$. This cavity is also defined by its opening (Lcav). The developed antenna must have a finite 
dimension in order to be easily implanted. The cavity is the first element to optimize. Its opening must be fixed in front. This structure shows a magnetic resonance at a frequency, the position of this resonance frequency can be varied by changing the different geometric parameters of the SRR, the largest dimension of the cell must be sufficiently small with respect to the wavelength $(\mathrm{d}<<\lambda)$.

\subsection{Antenna design}

The proposed antenna geometry and configuration is depicted in Figure 1. Where a threelayer structure consists of a substrate, air-gap, and a superstrate of thicknesses h1, H, and h3, respectively. The antenna is designed to operate at $5.8 \mathrm{GHz}$ for wireless application. The metamaterial surface is constructed on a dielectric material having a thickness of $1.6 \mathrm{~mm}$ and a dielectric constant of 2.2. This layer maintains a resonance distance of $0.5 \lambda$ (where $\lambda$ is the operating wavelength) above the substrate, the metamaterial surface is composed of $9 \times 9$-unit cells. The cell unit is a rectangular split ring resonator with a compact size of $X=Y=7 \mathrm{~mm}$. Each cell has a spacing of $0.5 \mathrm{~mm}$ in the $\mathrm{x}$-direction and $0.5 \mathrm{~mm}$ in the $\mathrm{y}$-direction, the width of the copper strip is $0.5 \mathrm{~mm}$. Considering the reference element the antenna is fed by a 50-Ohm coaxial probe form backside. The antenna has a rectangular radiation patch. The operation frequency of the patch antenna is inversely proportional to the length of its main radiator and can be configured by changing the antenna parameters.

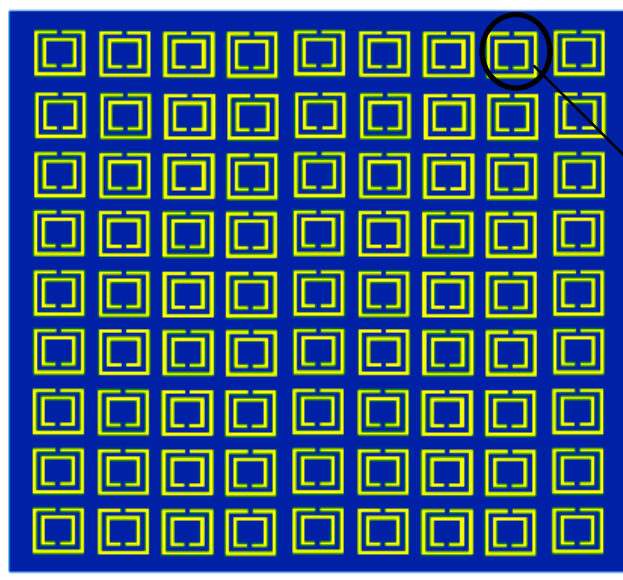

(a)

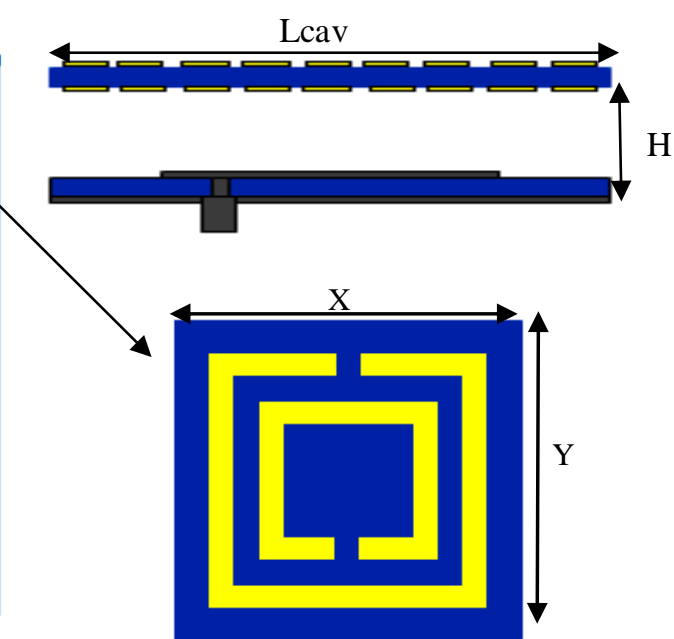

(b)

Fig. 1. Patch antenna with a double layer MMT layer. (a) top and and front view of the antenna (b)SRR unit cell. 


\section{Simulated results and discussion}

The reflexion coefficient versus frequency of the patch antenna with and without the double layer MTM superstrate is presented in Figure 2.the figure show that the patch antenna itself is not in a good matching condition compared when the double layer MTM superstrate has not been used. Strong resonance in cavity improves the matching and the resonant MTM antenna successfully and it operates over a reasonable band with a center frequency of $5.8 \mathrm{GHz}$. The double layer MTM antenna is in a good matching with a return loss of about $-33 \mathrm{~dB}$ at the resonance frequency $5.8 \mathrm{GHz}$ compared with the reference antenna alone which is $-12 \mathrm{~dB}$.

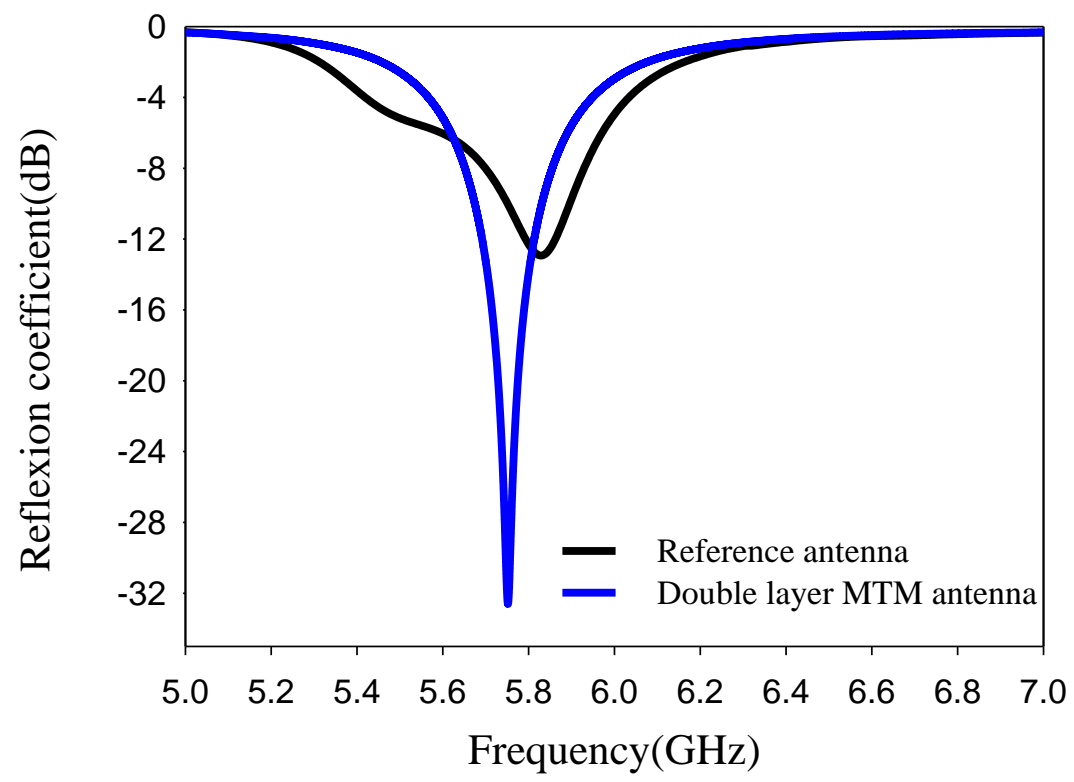

Fig. 2. reflection coefficient of the patch antenna with and without double layer MTM superstrate.

the directivity of the antenna with and without the double layer MTM superstrate is presented in Figure 3. It is seen that the directivity of the microstrip antenna covered by the double layer MTM superstrate is higher than that of the antenna without using the metasurface. The maximum directivity attained at the center frequency of $5.8 \mathrm{GHz}$ is $7.2 \mathrm{~dB}$ (without double layer metamaterial superstrate) and $14.1 \mathrm{~dB}$ (with double layer metamaterial superstrate) which is about $7 \mathrm{~dB}$ improvement. This shows that the proposed double layer MTM has a remarkable effect on the enhancement of the radiation energy, at an overall cavity height of $0.5 \lambda$.

The simulated 2D E-plane and H-plane radiation pattern of the double layer MTM superstrate loaded antenna in comparison to the reference antenna at the resonant frequency $5.8 \mathrm{GHz}$ are shown in Figure 4. We can observe that the main lobe is as expected in the direction of the 
positive z-axis (the direction of propagation) and that the back-lobe level decreases and broad side level increases by the loading of double layer MTM superstrate. It's clear from the plot that loading a microstrip antenna with MTM superstrate, has an important effect on the reduction of the $-3 \mathrm{~dB}$ beam width which degrade from $28,3^{\circ}$ to $104^{\circ}$ in the E plan and from $30,4^{\circ}$ to $83,5^{\circ}$ in the $\mathrm{H}$ plan ,the reduction in $3-\mathrm{dB}$ beam width is indication of the improved directivity in the broadside direction.

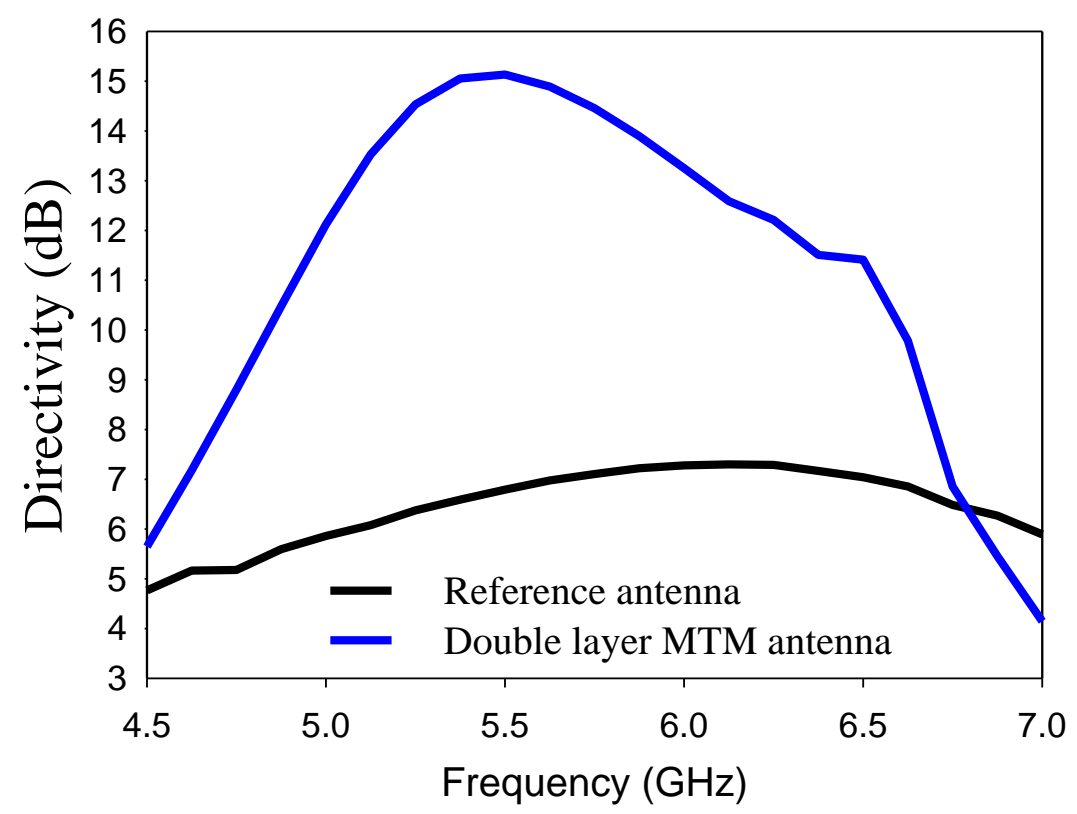

Fig. 3. Directivity of the patch antenna with and without double MTM superstrate. 


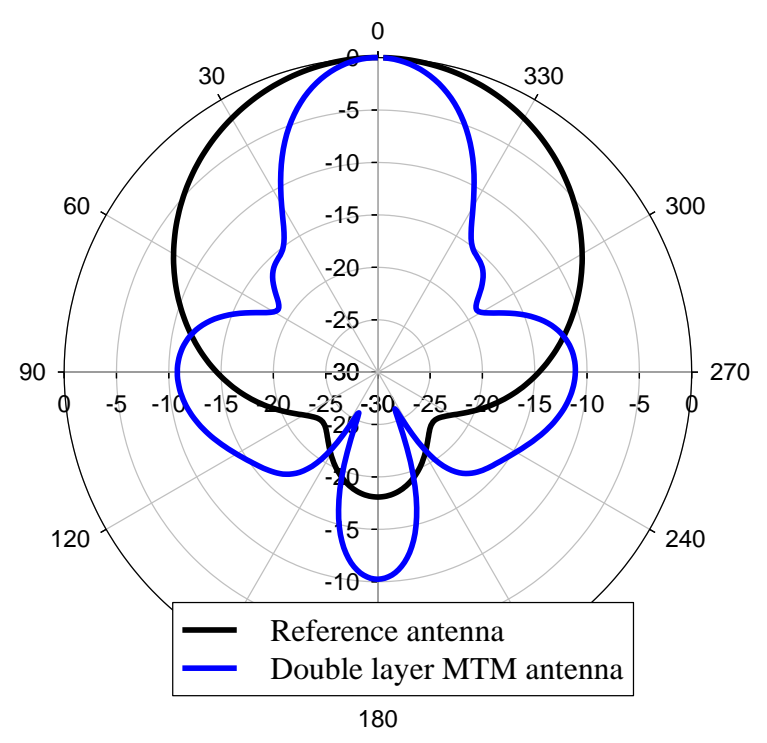

(a)

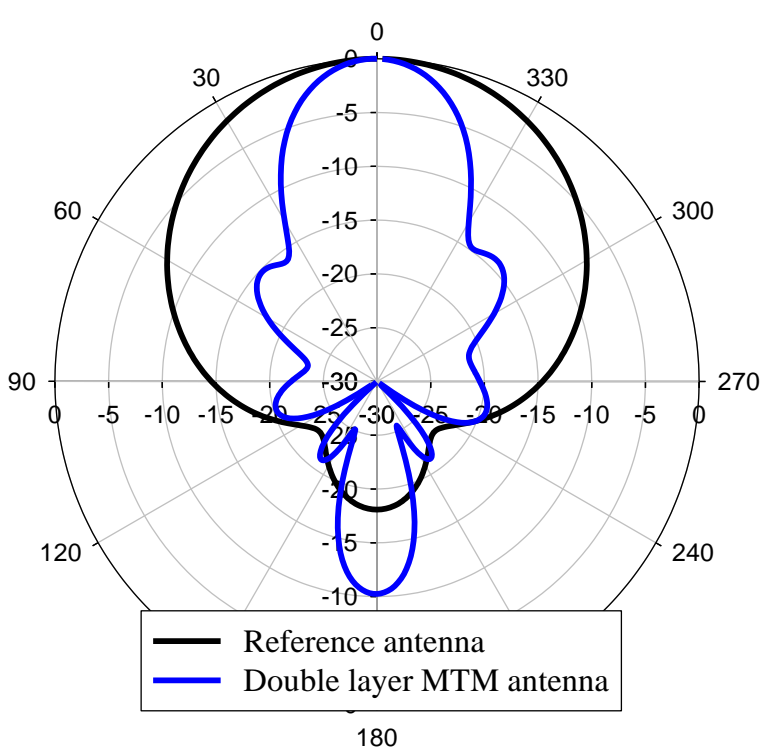

(b)

Fig. 4. Radiation pattern of designed antenna at $5.8 \mathrm{GHz}$ with and without the double layer MTM superstrate: (a) H-plane radiation pattern (b) E-plan radiation pattern 


\section{Conclusion}

The results obtained are encouraging, the structure allowed us to improve the directivity of the antenna patch within the frequency range of interest by a value of $7 \mathrm{~dB}$. this structure not only allows improving the directivity of the patch but also other characteristics, the double layer MTM antenna is in a good matching with a return loss of about $-33 \mathrm{~dB}$ at the resonance frequency $5.8 \mathrm{GHz}$ compared with the reference antenna alone which is $-12 \mathrm{~dB}$, The HPBW of the antenna with metasurface is narrowed down to $28.3^{\circ}$ from $104^{\circ}$ degree in the E plan and to $30.4^{\circ}$ from $83.5^{\circ}$ in the $\mathrm{H}$ plan,considering the radiation efficiency before and after using the metasurface it has been improved over the relevant range of frequencies from $70 \%$ to $83 \%$. This indicates that the double layer metamaterial superstrate added has a very important effect on antenna patch performance. In conclusion, the double layer MTM superstrate concentrates the radiation energy of the patch antenna and therefore it makes possible to improve certain characteristics of reference element, high directivity is the major demand of telecommunication and space applications. Hence the design of such antennas is the key requirement in order to overcome the restrictive parameters of the conventional antennas, we believe that similar idea can also be applied to other types of planar antennas and arrays.

\section{References}

[1] D.Nascimento, J. da, S. Lacava: Design of arrays of linearly polarized patch antennas on an FR-4 substrate. IEEE Antennas Propag Mag.pp. 12-22 (2015).

[2] X . Tang, H. Wong, Y. Long, Q . Xue, KL . Lau:Circularly polarized shorted patch antenna on high permittivity substrate with wideband. IEEE Trans Antennas Propag. pp. 1588-92 (2012).

[3] H. Nakano, M. Ikeda, K. Hitosugi, and Yamauchi : A spiral antenna sandwiched by dielectric layers. IEEE Trans. Antennas Propag.vo1. 52.no. 6 (2004).

[4] D. Li, Z. Szabo, X. Qing, E. P. Li, and Z. N. Chen : A high gain antenna with an optimized metamaterial inspired superstrate. IEEE Trans. Antennas Propag.vol. 60, no. 12, pp. 6018-6023 (2012).

[5]U.kumar, D. K. Upadhyay, B. L. Shahu :Improvement of performance parameters of rectangular patch antenna using metamaterial. IEEE International Conference On Recent Trends In Electronics Information Communication Technology. pp. 20-21 (2016).

[6]S. Gautam, A. Agrawal : Directivity Improvement of Patch Antenna using Metamaterial, Symposium on Colossal Data Analysis and Networking (CDAN). (2016).

[7]J. W. Jayasinghe, J. Anguera, D. N. Uduwaw: A high-directivity microstrip patch antenna design by using genetic algorithm optimization. Progress In Electromagnetics Research C, Vol. 37,131-144 (2013).

[8]Z. Xiao, J .Yao,S. Yin: High-Directivity Antenna Array Based on Artificial Electromagnetic Metamaterials with Low Refractive Index,Corporation International Journal of Antennas and Propagation, Hindawi Publishing. pp. 294-598 (2015).

[9]H. D. Yang, N. G. Alexopoulos , E. Yablonovitch :Photonic band-gap materials for high-gain printed circuit antennas. IEEE Trans Antennas and Propag, vol. 45, no. 1, pp. 185-187 (1997).

[10] B. Temelkuran, M. Bayindir, E. Ozbay et al: Photonic crystalbased resonant antenna with a very high directivity. Journal of Applied Physics. vol. 87, no. 1, pp. 603-605, (2000). 
[11] A. R. Weily, L. Horvath, K. P. Esselle, B. C. Sanders, and T. S. Bird : A planar resonator antenna based on a woodpile EBG material. IEEE Transactions on Antennas and Propagation. vol. 53, no. 1, pp. 216-223 (2005).

[12] S. Enoch, G. Tayeb, P. Sabouroux, N. Guerin, and P. Vincent : A metamaterial for directive emission. Physic Review Letters.vol. 89, no. 21, pp. 213902-1-213902-4 (2002).

[13]R Q. Wu, P. Pan, F.-Y. Meng, L.-W. Li, and J. Wu: A novel flat lens horn antenna designed based on zero refraction principle of metamaterials.Applied Physics : Materials Science and Processing.vol. 87, no. 2, pp. 151-156 (2007).

[14]J.B. Pendry: Negative Refraction Makes a Perfect Lens. Physical Review Lett 85, pp. 3966-3969 (2000).

[15]W. Cai, U. K. Chettiar, A. V. Kildishev, V. M. Shalaev : Optical cloaking with metamaterials .Nat.Photon 1, pp. 224 (2007).

[16]R. Marqués, F. Martín, and M. Sorolla, : Metamaterials with Negative Parameters: Theory, Design, and Microwave Application. John Wiley, New York (2008).

[17]J. B. Pendry, A.J. Holden, D.J. Robbins, and W. J. Stewart : Magnetism from conductors and enhanced nonlinear phenomena. IEEE Trans. Microwave Theory, Tech, 47 pp. 2075-2084 (1999).

[18]D. R. Smith, W. Padilla, D.C. Vier, S.C. Nemat-Nasser, S. Schultz : Composite Medium with Simultaneously Negative Permeability and Permittivity. Phys. Rev. Lett. 84 pp.4184 (2000). 